\title{
Adjunctive metformin for antipsychotic-induced dyslipidemia: a meta-analysis of randomized, double-blind, placebo-controlled trials
}

Wen-Long Jiang ${ }^{1}$, Dong-Bin Cai ${ }^{2}$, Fei Yin ${ }^{3}$, Ling Zhang ${ }^{4}$, Xi-Wu Zhao ${ }^{1}$, Jie He ${ }^{5}$, Chee H. Ng ${ }^{6}$, Gabor S. Ungvari ${ }^{7}$, Kang Sim $\mathbb{1 0}^{9}$, Mei-Ling Hu${ }^{10}$, Wei Zheng ${ }^{11}$ and Yu-Tao Xiang ${ }^{12}$

\begin{abstract}
Antipsychotic-induced dyslipidemia could increase the risk of cardiovascular diseases. This is a meta-analysis of randomized double-blind placebo-controlled trials to examine the efficacy and safety of adjunctive metformin for dyslipidemia induced by antipsychotics in schizophrenia. The standardized mean differences (SMDs) and risk ratios (RRs) with their 95\% confidence intervals (Cls) were calculated using the random-effects model with the RevMan 5.3 version software. The primary outcome was the change of serum lipid level. Twelve studies with 1215 schizophrenia patients (592 in metformin group and 623 in placebo group) were included and analyzed. Adjunctive metformin was significantly superior to placebo with regards to low density lipoprotein cholesterol (LDL-C) [SMD: -0.37 (95\%Cl:-0.69, $-0.05), P=0.02 ; P^{2}=78 \%$ ], total cholesterol [SMD: -0.47 (95\%Cl:-0.66, -0.29$), P<0.00001 ; P^{2}=49 \%$ ], triglyceride [SMD: -0.33 (95\%Cl:-0.45, -0.20$), P<0.00001 ; P=0 \%]$, and high density lipoprotein cholesterol [SMD: 0.29 (95\% Cl:0.02, 0.57), $P=0.03 ; P^{2}=69 \%$ ]. The superiority of metformin in improving LDL-C level disappeared in a sensitivity analysis and $80 \%(8 / 10)$ of subgroup analyses. Metformin was significantly superior to placebo with regards to decrease in body weight, body mass index, glycated hemoglobin A1c, fasting insulin, and homeostasis model assessment-insulin resistance $(P=0.002-0.01)$, but not regarding changes in waist circumference, waist-to-hip rate, leptin, fasting glucose, and blood pressure $(P=0.07-0.33)$. The rates of discontinuation due to any reason [RR: 0.97 (95\%Cl: $\left.0.66,1.43), P=0.89 ; P^{2}=0 \%\right]$ was similar between the two groups. Adjunctive metformin could be useful to improve total cholesterol and triglyceride levels, but it was not effective in improving LDL-C level in schizophrenia.
\end{abstract}

\section{Introduction}

Use of antipsychotics, particularly atypical antipsychotics, has been associated with a higher risk of metabolic and cardiovascular adverse effects ${ }^{1-3}$ and even premature death ${ }^{4,5}$, which could be significantly attributed to dyslipidemia ${ }^{6}$, particularly elevated low density lipoprotein cholesterol (LDL-C) $)^{7-9}$.

\footnotetext{
Correspondence: Wei Zheng (zhengwei0702@163.com) or

Yu-Tao Xiang (xyutly@gmail.com)

${ }^{1}$ The Third People's Hospital of Daqing, Daqing, China

${ }^{2}$ Shenzhen Traditional Chinese Medicine Hospital, Shenzhen, China

Full list of author information is available at the end of the article

There authors contributed equally: Wen-Long Jiang, Dong-Bin Cai, Fei Yin,

Ling Zhang
}

Dyslipidemia, such as increased level of total cholesterol, triglyceride, LDL-C and decreased level of high density lipoprotein cholesterol (HDL-C), could occur in around two-thirds of patients with schizophrenia ${ }^{10}$. Lifestyle and dietary interventions has been found to be safe and effective in treating dyslipidemia ${ }^{11,12}$. Several metaanalyses have found that topiramate ${ }^{12}$, metformin $^{13}$, and rosuvastatin ${ }^{14}$ were also effective and safe in improving dyslipidemia induced by antipsychotics in schizophrenia.

Metformin, a biguanide hypoglycemic agent, is widely prescribed for type 2 diabetes ${ }^{1}$. Animal studies have found metformin is effective in treating weight gain induced by olanzapine $^{15}$ and dyslipidemia induced by risperidone ${ }^{16}$. Case reports and open-label studies also found that 
metformin could improve body weight ${ }^{17}$ and metabolic syndrome ${ }^{17}$. However, the findings of randomized doubleblind placebo-controlled trials (RCTs) ${ }^{6,18-28}$ examining the effects of adjunctive metformin for dyslipidemia induced by antipsychotics have been mixed.

Some reviews and meta-analyses ${ }^{11,13,29-37}$ have examined the efficacy and safety of adjunctive metformin for antipsychotics-related weight gain and metabolic abnormalities in schizophrenia. However, the primary outcome in most meta-analyses was weight gain, rather than dyslipidemia, and some meta-analyses included open-label studies, which deviate from the standardized recommendations ${ }^{38}$. In addition, the findings of the previous meta-analyse ${ }^{12,13,33,35,37}$ on adjunctive metformin for dyslipidemia, such as total cholesterol, HDL and LDL cholesterol, have been inconsistent. Thus, we conducted this meta-analysis of RCTs in English and Chinese literature to examine the efficacy and safety of adjunctive metformin for dyslipidemia induced by antipsychotics in schizophrenia.

\section{Methods}

\section{Search strategy}

Both international (PubMed, Cochrane Library, PsycINFO, and EMBASE) and Chinese (Chinese Journal Net, and WanFang) databases were independently searched to obtain relevant published RCTs by three investigators (WLJ, DBC, and FY) from their commencement dates to October 22, 2018, using the search terms as follows: ("metformin" [MeSH] OR metformin OR Dimethylbiguanidine OR Dimethylguanylguanidine OR Glucophage) AND ("dyslipidemias"[MeSH] OR Dyslipidemias OR Dyslipidemia OR Dyslipoproteinemias OR Dyslipoproteinemia OR metabolic OR lipid OR fats OR Hypolipidemic Agents OR cholesterol OR HDL OR LDL OR lipoprotein OR triglyceride OR adiponectin OR ghrelin OR leptin OR resistin OR chemerin OR omentin OR apelin or adipocytokine OR adipokine) AND ("schizophrenia"[Mesh] OR schizophrenic disorder OR disorder, schizophrenic OR schizophrenic disorders OR schizophrenia OR dementia praecox). The references of relevant reviews ${ }^{11,13,29-37}$ were also searched manually to avoid missing publications.

\section{Inclusion criteria of the meta-analysis}

The inclusion criteria of this meta-analysis were made according to PICOS acronym. Participants: patients with schizophrenia diagnosed according to international or local diagnostic criteria, such as the China's mental disorder classification and diagnosis standard, 3rd edition, International Classification of Diseases, 10th edition or Diagnostic and Statistical Manual of Mental Disorders, 4th edition. Intervention: adjunctive metformin with treatment as usual (TAU). Comparison: TAU plus placebo. Outcomes: the primary outcome was LDL-C $(\mathrm{mg} / \mathrm{dL})$; the secondary outcomes were total cholesterol $(\mathrm{mg} / \mathrm{dL})$, triglyceride $(\mathrm{mg} / \mathrm{dL})$, HDL-C $(\mathrm{mg} / \mathrm{dL})$, body weight $(\mathrm{kg})$, body mass index $\left(\mathrm{BMI}, \mathrm{kg} / \mathrm{m}^{2}\right)$, waist circumference $(\mathrm{cm})$, waist-to-hip rate, leptin $(\mathrm{ug} / \mathrm{L})$, glycated hemoglobin A1c (HbA1c, \%), fast insulin (mIU/L) and homeostasis model assessment of insulin resistance (HOMA-IR), fasting glucose $(\mathrm{mmol} / \mathrm{L})$, blood pressure including diastolic and systolic blood pressure $(\mathrm{mmHg})$, adverse drug reactions (ADRs), and discontinuation due to any reason. Study: following other meta-analyses ${ }^{39,40}$, only published double-blind RCTs that examined the efficacy and safety of metformin for dyslipidemia with available data were included. Studies without data of blood lipid were excluded ${ }^{41-43}$.

\section{Data extraction}

Three investigators (WLJ, DBC, and FY) independently extracted relevant data of eligible studies, including study characteristics (such as first author, publication year and sample size), basic demographic and clinical data (such as age, percentage of males, diagnostic criteria, illness duration, antipsychotic and its doses) and outcomes (efficacy, safety and tolerability of metformin). Discrepancies in literature search and data extraction were resolved through negotiation, or consultations with a senior investigator (WZ). One study $^{19}$ with 2 active treatment arms compared two different doses of metformin with placebo. The data of these three groups (2 active treatment arms +1 placebo group) were separately extracted and analyzed in this meta-analysis. In order to avoid inflating the total number of patients in the placebo group, we assigned half of those in the placebo group to each metformin arm.

\section{Statistical analyses}

The data analyses were performed by the RevMan, version 5.3 according to the recommendations of the Cochrane Collaboration ${ }^{44}$. The random-effects model was used for all meta-analyzable outcomes ${ }^{45}$. The standardized mean differences (SMDs) and their 95\% confidence intervals (CIs) were calculated for continuous outcomes. The risk ratios (RRs) and their 95\% CIs were calculated for categorical outcomes. Significant heterogeneity was defined by $I^{2}$ of $>50 \%$ or $P$ value of $<0.1$ in Q-test.

In case of $I^{2} \geq 50 \%$ for LDL-C level, a sensitivity analysis was conducted to detect the source of heterogeneity after removing 1 outlying study $(\mathrm{SMD} \leq-0.87)^{18}$. Furthermore, the following six subgroup analyses were conducted to identify the sources of heterogeneity of the primary outcome: (i) Chinese vs. non-Chinese studies; (ii) studies with olanzapine vs. studies that did not use olanzapine as the primary antipsychotic medication; (iii) trial duration (weeks): $\geq 16$ vs. $<16$; (iv) no sex predominance vs. male 
predominance ( $\geq 60 \%$ ); (v) high quality (Jadad score $\geq 3$ ) vs. low quality (Jadad score $<3$ ); and (vi) age (years): $\geq 39.3$ vs. $<39.3$ (using the mean split of age). Sensitivity and subgroup analyses were not performed for total cholesterol and triglyceride levels, as the heterogeneity was small $\left(I^{2}=49 \%\right.$ and $0 \%$, respectively). Publication bias was examined by visual funnel plots and Egger's test ${ }^{46}$ using comprehensive meta-analysis program version 2. All meta-analytic primary and secondary outcomes were two tailed, with alpha set at 0.05 .

\section{Assessment of study quality}

The quality of included RCTs was independently assessed by three investigators (WLJ, DBC, and FY) using Jadad scale ${ }^{47,48}$ and the Cochrane Risk of bias ${ }^{49}$. The overall evidence levels of all meta-analytic outcomes were measured using the grading of recommendations, assessment, development, and evaluation (GRADE) system $^{50,51}$.

\section{Results}

\section{Computer search}

A total of 454 hits (Fig. 1) were identified from the databases $(n=451)$ and manual search $(n=3)$. Finally, 12 studies with $13 \mathrm{RCTs}^{6,18-28}$ were included in this meta-analysis. Of them, one study contained two RCTs ${ }^{6}$.

\section{Study characteristics}

The 12 studies with 13 RCTs covered 1215 patients (592 in the metformin group (500-2550 mg/day) and 623 in the placebo group; Table 1). The mean age was 39.3 (range $=26.0-47.7$ ) years and the mean illness duration in 11 studies with available data was 10.7 (range $=0.5-24.8$ ) years. The mean trial duration was 15.5 (range $=8-24$ ) weeks. Eight RCTs were conducted in China $(n=826)$, three in Venezuela $(n=181)$, and one each in Iran $(n=60)$ and the USA $(n=148)$.

\section{Quality assessment of included study}

Of the 13 RCTs, only $1 \mathrm{RCT}^{22}$ was rated as high risk with regards to the random sequence generation and allocation concealment (Supplemental Fig. 1). Other bias was rated as unclear in all RCTs. The Jadad total scores ranged from 2 to 5 ; 11 RCTs were rated as high quality (Jadad score $\geq 3$ ). The quality of evidence for primary and secondary outcomes using the GRADE approach ranged from "low" (8.4\%, 2/24), to "moderate" $(45.8 \%, 11 / 24)$, and "high" (45.8\%, 11/24) (Supplemental Table 1).

\section{Serum lipid}

Adjunctive metformin was significantly superior to placebo with regard to LDL-C level [SMD: $-0.37(95 \% \mathrm{CI}$ : $\left.-0.69,-0.05), P=0.02 ; I^{2}=78 \%\right]$, total cholesterol level [SMD: $\quad-0.47 \quad(95 \%$ CI:-0.66, $\quad-0.29), \quad P<0.00001$;

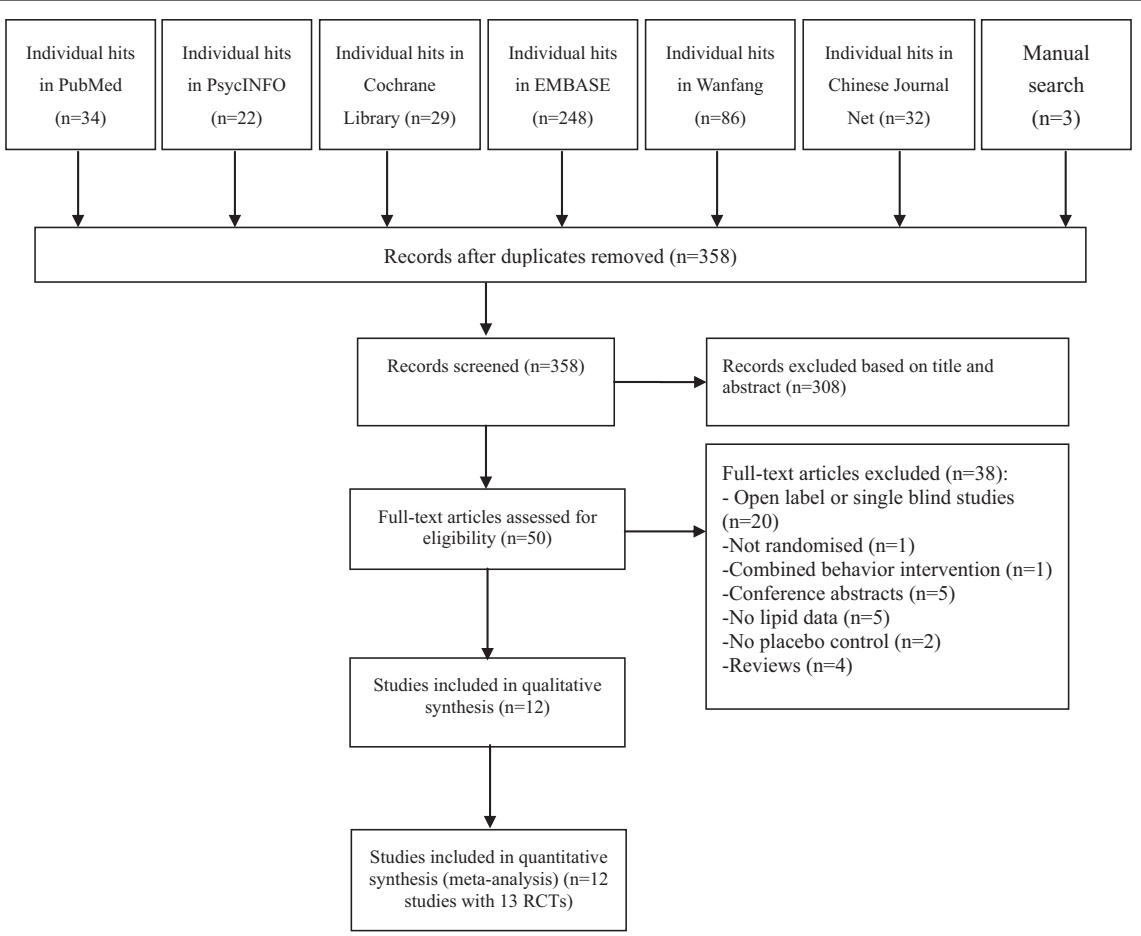

Fig. 1 Flow chart of literature search and study selection. This figure described the route of studies inclusion. 


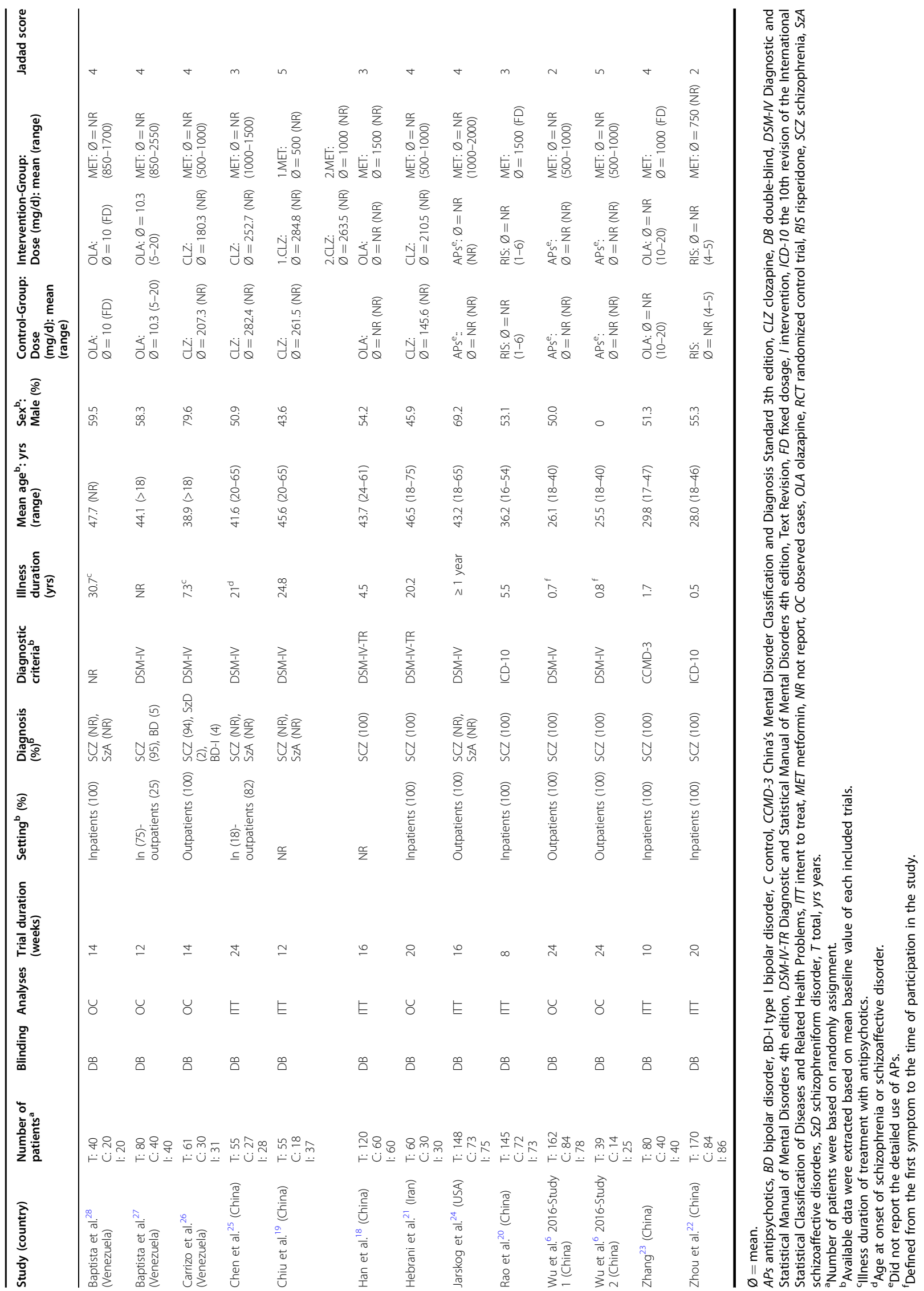


$I^{2}=49 \%$ ], triglyceride level [SMD: -0.33 (95\%CI:-0.45, $-0.20), P<0.00001 ; I^{2}=0 \%$ ], and HDL-C level [SMD: 0.29 (95\%CI:0.02, 0.57), $P=0.03 ; I^{2}=69 \%$ ] (Fig. 2).

The significance with regard to LDL-C level disappeared [SMD: -0.28 (95\%CI: $-0.61,0.05), P=0.10 ; I^{2}=75 \%$ ] after 1 outlying $(\mathrm{SMD} \leq-0.87)$ study $^{18}$ was removed. Similarly, the significance disappeared in 8 of the 10 subgroup analyses (Table 2).

\section{Anthropometric variables}

Metformin was significantly superior to placebo with regards to decrease in body weight [SMD: -0.35 (95\%CI: $-0.59,-0.11), P=0.005 ; I^{2}=69 \%$ ], BMI [SMD: -0.39 (95\%CI: $\left.-0.66,-0.12), P=0.005 ; I^{2}=76 \%\right]$, but not regarding waist circumference [SMD: $-0.15 \quad(95 \% \mathrm{CI}$ : $\left.-0.45,0.15), P=0.33 ; I^{2}=71 \%\right]$, and waist-to-hip rate [SMD: -0.46 (95\%CI: $-1.19,0.27), P=0.22 ; I^{2}=91 \%$, leptin [SMD: -1.09 (95\%CI: $-2.26,0.07), P=0.07 ; I^{2}=$ 94\%] (Table 3).

\section{Carbohydrate metabolism}

Metformin was significantly superior to placebo with regards to HbA1c [SMD: -0.32 (95\%CI:-0.52, -0.12 ), $P=0.002 ; I^{2}=0 \%$ ], fasting insulin [SMD: -0.73 (95\%CI: $-1.22,-0.24), P=0.003 ; I^{2}=87 \%$ ] and HOMA-IR [SMD: -0.89 (95\%CI:-1.57, -0.21$\left.), P=0.01 ; I^{2}=92 \%\right]$, but not regarding fasting glucose [SMD: $-0.31(95 \% \mathrm{CI}:-0.67$, 0.04), $P=0.09 ; I^{2}=88 \%$ ] (Table 3 ).

\section{Blood pressure}

No significant differences were found regarding diastolic [SMD: -0.11 (95\%CI: $\left.-0.31,0.10), P=0.30 ; I^{2}=0 \%\right]$ and systolic blood pressure [SMD: -0.15 (95\%CI: $-0.35,0.06)$, $P=0.16 ; I^{2}=0 \%$ ] between two groups (Table 3 ).

\section{Adverse drug reactions and discontinuation rate}

Compared with placebo, adjunctive metformin was significantly associated with more frequent nausea/ vomiting $(P=0.02,95 \% \mathrm{CI}=11-50)$. No significant group differences were found in other ADRs $(P=0.14-0.98)$ and discontinuation due to any reason [RR: 0.97 (95\%CI: 0.66, 1.43), $P=0.89 ; I^{2}=0 \%$ ( Table 3 ).

\section{Publication bias}

The funnel plots of included studies were symmetrical and Egger's test did not detect publication bias for triglyceride level $(P=0.50)$, body weight $(P=0.50)$, and fasting glucose $(P=0.97)$. Publication bias of other metaanalytic outcomes could not be examined due to small numbers $(n<10)$ of RCTs ${ }^{52}$.

\section{Discussion}

This meta-analysis found that adjunctive metformin was effective, safe, and generally well-tolerated in treating total cholesterol and triglyceride levels with "high" evidence level assessed by the GRADE approach within schizophrenia patients for antipsychotic-induced dyslipidemia, which is consistent with the findings in studies involving several other adjunctive medications, such as rosuvastatin (total cholesterol level vs. triglyceride level: $\mathrm{SMD}=-2.00$ vs. -1.05$)^{14}$. However, the significant superiority of metformin over placebo in the improvement of LDL-C level was driven by an outlying study ${ }^{18}$. Similarly, metformin was not effective in improving LDL$\mathrm{C}$ level in 8 of the 10 subgroup analyses. This metaanalysis found the significant superiority of adjunctive metformin over placebo regarding LDL-C, total cholesterol, triglyceride and HDL-C level with small effect sizes $^{53}$. Convincing evidence showed that findings with small effect size could be also reliable in clinical practice. For instance, a recent network meta-analysis that compared the efficacy and acceptability of 21 antidepressant drugs for major depressive disorder found that all antidepressants were more efficacious than placebo in improving depressive symptoms, but only small effect sizes were achieved ${ }^{54}$.

The mechanism of metformin underlying the treatment of antipsychotic-inducted dyslipidemia is still not clear. One possible explanation may be related to the role of metformin in improving insulin resistance ${ }^{6,19,33,35}$ which is a risk factor of dyslipidemia ${ }^{55}$ and could decrease lipoprotein synthesis ${ }^{56}$. In addition, the therapeutic effects of metformin in treating antipsychotic-inducted dyslipidemia may be associated with its role in the neuronal reduction of endogenous glucose production, increasing glucagon-like peptide-1 production, decreasing bile acid concentration in enterocytes, and modulating the gut microbiota ${ }^{56}$.

In this meta-analysis, adjunctive metformin showed significant benefits for reducing body weight and BMI, which is consistent with the findings of other metaanalysis $^{13,57}$. For example, a recent meta-analysis found that a combination of metformin and lifestyle modification could significantly reduce body weight and BMI compared with placebo, lifestyle group or metformin alone $^{11}$. Adjunctive metformin for antipsychotic-induced dyslipidemia were generally safe and well-tolerated. In this meta-analysis, seven studies reported more frequent nausea/vomiting in adjunctive metformin compared with placebo. A meta-analysis of use of adjunctive metformin for antipsychotic-induced weight gain also found that metformin could lead to more frequent reports of nausea/ vomiting than placebo ${ }^{13}$.

Some studies found that long-term use of metformin was associated with decreased vitamin $B_{12}$ levels, and even biochemical $\mathrm{B}_{12}$ deficiency ${ }^{2,58}$. Thus, blood lactate concentration, serum $B_{12}$ levels and folate levels should be regularly screened for patients with schizophrenia when they received metformin treatment ${ }^{2}$. 


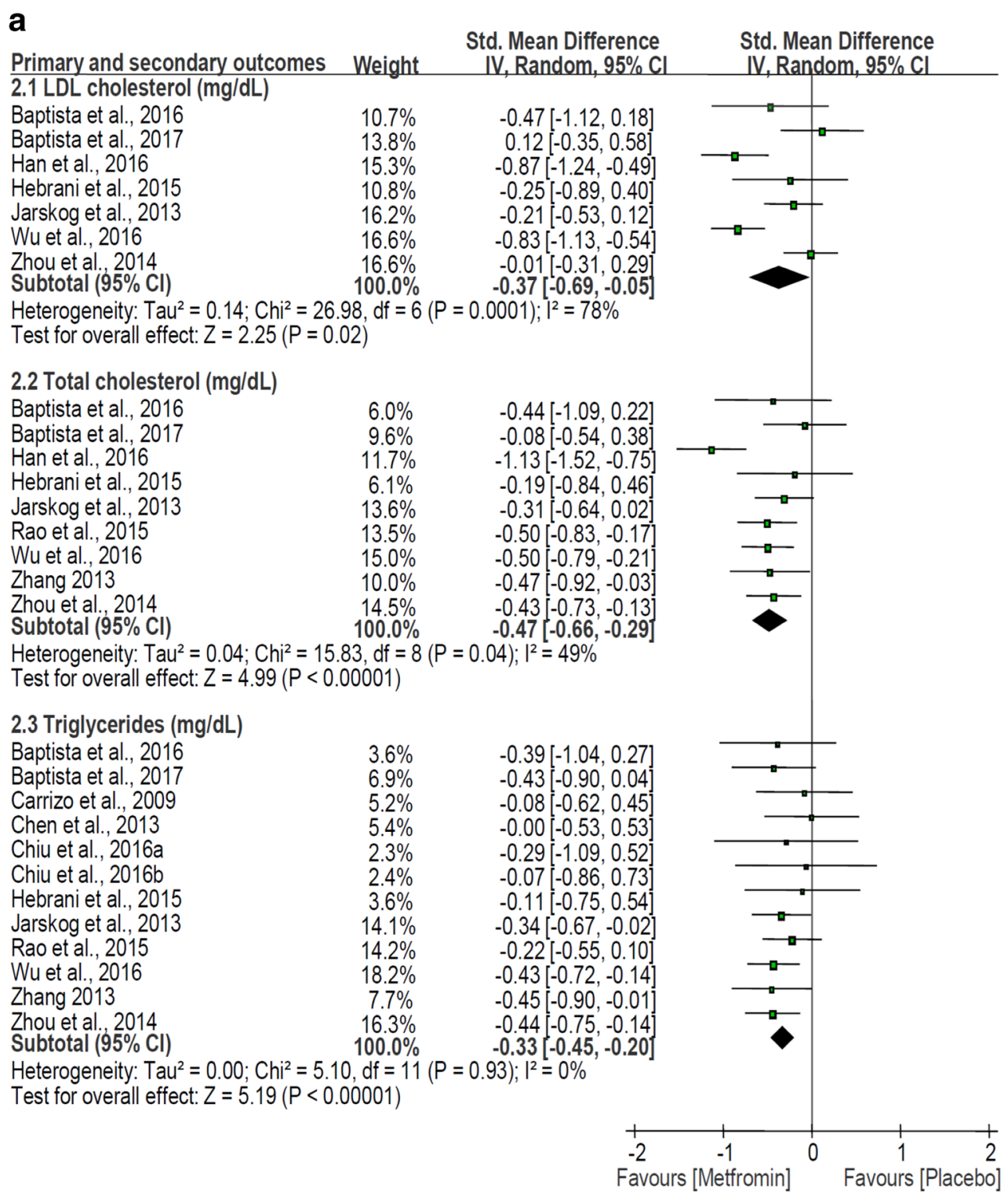

\section{b}

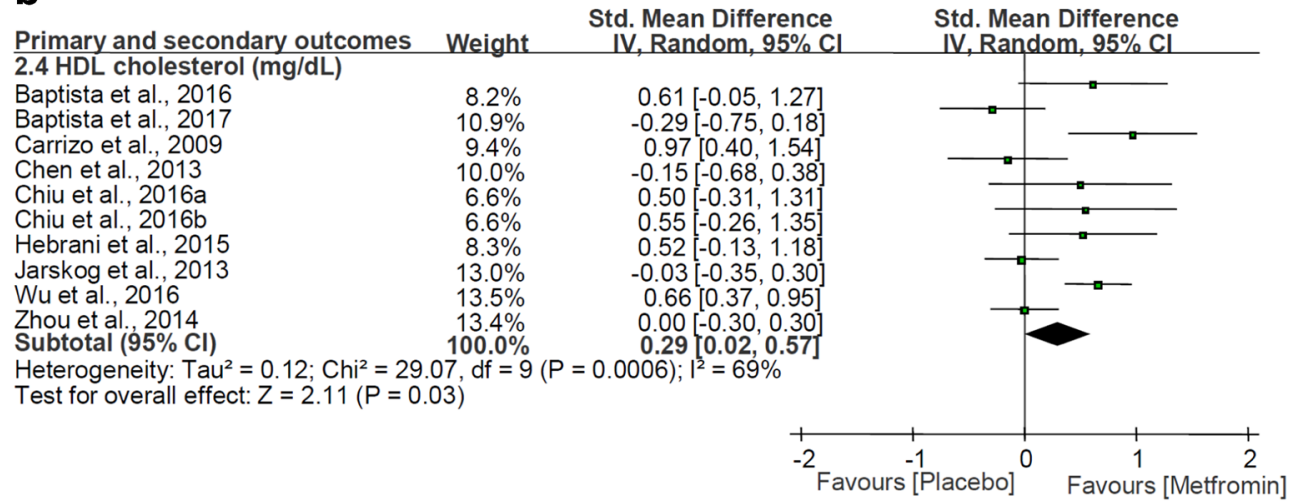

Fig. 2 Adjunctive metformin for antipsychotic-induced dyslipidemia: forest plot for serum lipid. Adjunctive metformin was significantly superior to placebo with regard to LDL-C level, total cholesterol level, triglyceride level, and HDL-C level. HDL-C high density lipoprotein cholesterol, LDL-C low density lipoprotein cholesterol. 
Table 2 Subgroup analyses of metformin for LDL-C level.

\begin{tabular}{|c|c|c|c|c|}
\hline Variables & $\begin{array}{l}\text { Active arms } \\
\text { (subjects) }\end{array}$ & SMDs $(95 \% \mathrm{Cl})$ & $I^{2}(\%)$ & $\mathbf{P}$ \\
\hline 1. Chinese & $3(480)$ & $-0.57(-1.13,-0.00)$ & 89 & 0.05 \\
\hline Non-Chinese & $4(292)$ & $-0.16(-0.39,0.07)$ & 0 & 0.16 \\
\hline $\begin{array}{l}\text { 2. Antipsychotic } \\
\text { class: olanzapine }\end{array}$ & $3(229)$ & $-0.41(-1.06,0.23)$ & 81 & 0.21 \\
\hline $\begin{array}{l}\text { Other than } \\
\text { olanzapine }\end{array}$ & $4(543)$ & $-0.33(-0.75,0.08)$ & 81 & 0.11 \\
\hline $\begin{array}{l}\text { 3. Trial duration } \\
\text { (weeks): } \geq 16\end{array}$ & $5(663)$ & $-0.44(-0.82,-0.06)$ & 82 & 0.02 \\
\hline$<16$ & $2(109)$ & $-0.13(-0.70,0.44)$ & 51 & 0.66 \\
\hline 4. Jadad score ${ }^{a} \geq 3$ & $6(602)$ & $-0.44(-0.78,-0.10)$ & 74 & 0.01 \\
\hline Jadad score $<3$ & $1(170)$ & $-0.01(-0.31,0.29)$ & N/A & 0.93 \\
\hline $\begin{array}{l}\text { 5. Age (years) }{ }^{a} \text { : } \\
\geq 39.3\end{array}$ & $5(412)$ & $-0.34(-0.70,0.02)$ & 67 & 0.07 \\
\hline$<39.3$ & $2(360)$ & $-0.42(-1.23,0.38)$ & 93 & 0.30 \\
\hline
\end{tabular}

Bold $P$ values: $P<0.05$.

Cl confidence interval, N/A not applicable, $L D L-C$ low density lipoprotein cholesterol, SMDs standard mean differences.

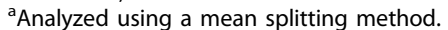

Several meta-analyses ${ }^{11,13,29-37}$ had examined antipsychotic-induced body weight and metabolic abnormalities in schizophrenia, however, only a small number of them with comparatively smaller sample size meta-analyzed dyslipidemia as a secondary outcome ${ }^{33,35,37}$. This meta-analysis only included double-blind RCTs examining adjunctive metformin for dyslipidemia in schizophrenia, together with a large sample size increase the robustness and reliability of meta-analytic findings.

Several limitations should be noted. First, the significant heterogeneity for primary outcome remained, even in some subgroup analyses. For this reason, we tried to compensate for study heterogeneity using the randomeffects model and conducting subgroup analyses. Second, although unpublished studies were not searched, no publication bias was found for triglyceride level, body weight, and fasting glucose in this meta-analysis. Third, the dose-response effect of metformin as an adjunctive treatment for dyslipidemia was not examined since the doses of metformin varied across included RCTs (500-2550 mg/day). Fourth, the significant superiority of metformin over placebo in improving LDL-C level was found in RCTs lasting more than 16 weeks, but not in those less than 16 weeks. Furthermore, the long-term effect (beyond 24 weeks) of adjunctive metformin for antipsychotic-induced dyslipidemia was not examined.

In conclusion, adjunctive metformin could improve total cholesterol and triglyceride levels but its therapeutic effects on LDL-C level was less certain in this meta-
Table 3 Adjunctive metformin for antipsychotic-induced dyslipidemia: secondary outcomes.

\begin{tabular}{|c|c|c|c|c|}
\hline Variables & $\begin{array}{l}\text { Active arms } \\
\text { (subjects) }\end{array}$ & SMDs/RRs $(95 \% \mathrm{Cl})$ & $I^{2}(\%)$ & $P$ \\
\hline \multicolumn{5}{|l|}{ Clinical features } \\
\hline Body weight (kg) & $11(1004)$ & $-0.35(-0.59,-0.11)$ & 69 & 0.005 \\
\hline $\mathrm{BMI}\left(\mathrm{kg} / \mathrm{m}^{2}\right)$ & $12(1016)$ & $-0.39(-0.66,-0.12)$ & 76 & 0.005 \\
\hline $\begin{array}{l}\text { Waist } \\
\text { circumference }(\mathrm{cm})\end{array}$ & $9(681)$ & $-0.15(-0.45,0.15)$ & 71 & 0.33 \\
\hline WHR & $3(346)$ & $-0.46(-1.19,0.27)$ & 91 & 0.22 \\
\hline Leptin (ug/L) & $3(238)$ & $-1.09(-2.26,0.07)$ & 94 & 0.07 \\
\hline $\begin{array}{l}\text { Fasting glucose } \\
(\mathrm{mmol} / \mathrm{L})\end{array}$ & $13(1161)$ & $-0.31(-0.67,0.04)$ & 88 & 0.09 \\
\hline $\mathrm{HbA1c}(\%)$ & $4(384)$ & $-0.32(-0.52,-0.12)$ & 0 & 0.002 \\
\hline Fast insulin (mIU/L) & $5(615)$ & $-0.73(-1.22,-0.24)$ & 87 & 0.003 \\
\hline HOMA-IR & $6(501)$ & $-0.89(-1.57,-0.21)$ & 92 & 0.01 \\
\hline $\begin{array}{l}\text { Diastolic blood } \\
\text { pressure }(\mathrm{mmHg})\end{array}$ & $5(371)$ & $-0.11(-0.31,0.10)$ & 0 & 0.30 \\
\hline $\begin{array}{l}\text { Systolic blood } \\
\text { pressure }(\mathrm{mmHg})\end{array}$ & $5(371)$ & $-0.15(-0.35,0.06)$ & 0 & 0.16 \\
\hline \multicolumn{5}{|l|}{ Discontinuation rate } \\
\hline $\begin{array}{l}\text { Discontinuation due } \\
\text { to any reason }\end{array}$ & $9(806)$ & $0.97(0.66,1.43)$ & 0 & 0.89 \\
\hline \multicolumn{5}{|l|}{ ADRs } \\
\hline Nausea/vomiting & $7(765)$ & $1.51(1.05,2.16)$ & 0 & 0.02 \\
\hline Dizziness & $2(315)$ & $1.99(0.56,7.06)$ & 0 & 0.29 \\
\hline Dry mouth & $4(596)$ & $1.76(0.82,3.76)$ & 0 & 0.14 \\
\hline Hypersomnia & $2(371)$ & $0.45(0.02,11.25)$ & 77 & 0.63 \\
\hline Tachycardia & $2(315)$ & $0.86(0.26,2.83)$ & 16 & 0.80 \\
\hline Headache & $2(316)$ & $1.02(0.17,6.05)$ & 44 & 0.98 \\
\hline Constipation & $3(395)$ & $1.23(0.52,2.88)$ & 10 & 0.64 \\
\hline Diarrhea & $5(394)$ & $1.43(0.86,2.41)$ & 19 & 0.17 \\
\hline
\end{tabular}

Bold $P$ values: $P<0.05$

$A D R s$ adverse drug reactions, $B M I$ body mass index, $\mathrm{Cl}$ confidence interval, $\mathrm{HbAlc}$ glycated hemoglobin A1C, HOMA-IR homeostasis model assessment-insulin resistance, $R R s$ risk ratios, SMDs standardized mean differences, WHR waist-tohip ratio.

analysis. The prolonged effect of active agents such as metformin to treat dyslipidemia induced by antipsychotics in schizophrenia needs to be further examined.

\section{Acknowledgements}

The study was supported by the National Key Research and Development Plan "Precision Medical Research" 2016 Project (2016YFC0906302) and Science and Technology Program of Guangzhou (201807010064).

\section{Author details}

${ }^{1}$ The Third People's Hospital of Daqing, Daqing, China. ${ }^{2}$ Shenzhen Traditional Chinese Medicine Hospital, Shenzhen, China. ${ }^{3}$ Harbin Medical University Daqing Campus, Daqing, China. ${ }^{4}$ The Third Affiliated Hospital of Sun Yat-Sen University, Guangzhou, China. ${ }^{5}$ The National Clinical Research Center for Mental Disorders \& Beijing Key Laboratory of Mental Disorders Beijing Anding Hospital \& the Advanced Innovation Center for Human Brain Protection, Capital Medical University, School of Mental Health, Beijing, China.

${ }^{6}$ Department of Psychiatry, The Melbourne Clinic and St Vincent's Hospital, University of Melbourne, Richmond, Victoria, Australia. ${ }^{7}$ University of Notre Dame Australia, Fremantle, Australia. ${ }^{8}$ Division of Psychiatry, School of Medicine, University of Western Australia, Perth, Australia. 'West Region, Institute of Mental Health, Buangkok Green Medical Park, Singapore, Singapore. ${ }^{10}$ The Fifth People's Hospital of Shangrao, Shangrao, China. ${ }^{11}$ The Affiliated Brain Hospital of Guangzhou Medical University (Guangzhou Huiai Hospital), 
Guangzhou, China. ${ }^{12}$ Unit of Psychiatry, Institute of Translational Medicine, Faculty of Health Sciences, University of Macau, Macao, SAR, China

\section{Conflict of interest}

The authors declare that they have no conflict of interest.

\section{Publisher's note}

Springer Nature remains neutral with regard to jurisdictional claims in published maps and institutional affiliations.

Supplementary Information accompanies this paper at (https://doi.org/ 10.1038/s41398-020-0785-y).

Received: 6 August 2019 Revised: 24 February 2020 Accepted: 20 March 2020

Published online: 23 April 2020

\section{References}

1. Lazarus, B. et al. Association of metformin use with risk of lactic acidosis across the tange of kidney function: a community-based cohort study. JAMA Intern. Med. 178, 903-910 (2018).

2. Andrade, C. Use of metformin for cardiometabolic risks in psychiatric practice: need-to-know safety issues. J. Clin. Psychiatry 77, e1491-e1494 (2016).

3. Meyer, J. M. \& Stahl, S. M. The metabolic syndrome and schizophrenia. Acta Psychiatr. Scand. 119, 4-14 (2009).

4. Boules, M., Fredrickson, P. \& Richelson, E. Neurotensin agonists as an alternative to antipsychotics. Expert Opin. Investig. Drugs 14, 359-369 (2005).

5. Reininghaus, U. et al. Mortality in schizophrenia and other psychoses: a 10-year follow-up of the SOP first-episode cohort. Schizophr. Bull. 41, 664-673 (2015).

6. Wu, R. R. et al. Metformin treatment of antipsychotic-induced dyslipidemia: an analysis of two randomized, placebo-controlled trials. Mol. Psychiatry 21, 1537-1544 (2016).

7. De Hert, M. et al. Treatment with rosuvastatin for severe dyslipidemia in patients with schizophrenia and schizoaffective disorder. J. Clin. Psychiatry 67, 1889-1896 (2006)

8. Hanssens, L. et al. Pharmacological treatment of severe dyslipidaemia in patients with schizophrenia. Int. Clin. Psychopharmacol. 22, 43-49 (2007).

9. Graham, I., Cooney, M. T., Bradley, D., Dudina, A. \& Reiner, Z. Dyslipidemias in the prevention of cardiovascular disease: risks and causality. Curr. Cardiol. Rep. 14, 709-720 (2012).

10. Shafie, S. et al. Prevalence and correlates of diabetes mellitus and dyslipidaemia in a long-stay inpatient schizophrenia population in Singapore. Singap. Med. J. 59, 465-471 (2018).

11. Zheng, W. et al. Combination of metformin and lifestyle intervention for antipsychotic-related weight gain: a meta-analysis of randomized controlled trials. Pharmacopsychiatry 52, 24-31 (2019).

12. Vancampfort, D. et al. The impact of pharmacological and nonpharmacological interventions to improve physical health outcomes in people with schizophrenia: a meta-review of meta-analyses of randomized controlled trials. World Psychiatry 18, 53-66 (2019).

13. Zheng, W. et al. Metformin for weight gain and metabolic abnormalities associated with antipsychotic treatment: meta-analysis of randomized placebo-controlled trials. J. Clin. Psychopharmacol. 35, 499-509 (2015).

14. Zheng, W. et al. Meta-analysis of the efficacy and safety of adjunctive rosuvastatin for dyslipidemia in patients with schizophrenia. Shanghai Arch. Psychiatry 30, 4-11 (2018).

15. Hu, Y. et al. Metformin and berberine prevent olanzapine-induced weight gain in rats. PLOS ONE9, e93310 (2014).

16. Adeneye, A. A., Agbaje, E. O. \& Olagunju, J. A. Metformin: an effective attenuator of risperidone-induced insulin resistance hyperglycemia and dyslipidemia in rats. Indian J. Exp. Biol. 49, 332-338 (2011).

17. Vishnupriya, R., Ezhilramya, J. \& Meenakshi, B. Metformin in the prevention of metabolic syndrome associated with initiation of atypical antipsychotic therapy in adolescents and young adults-a randomized, open labelled, single centered study. Int. J. Pharm. Pharm. Sci. 8, 200-206 (2016).

18. Han, X. L., Jia, J. M., Huang, T. X., Zhang, Z. \& Wang, F. H. A comparative study of metformin to improve glucose and lipid metabolism in schizophrenic patients with metabolic syndrome (In Chinese). J. Psychiatry 29, 443-447 (2016).

19. Chiu, C. C. et al. Effects of low dose metformin on metabolic traits in clozapine-treated schizophrenia patients: an exploratory twelve-week randomized, double-blind, placebo-controlled study. PLOS ONE 11, e0168347 (2016).

20. Rao, S. X. et al. Efficacy analysis of metformin combined with risperidone in the treatment of schizophrenia with metabolic syndrome (In Chinese). Neural Inj. Funct. Reconstr. 10, 358-359 (2015).

21. Hebrani, P. et al. Double-blind, randomized, clinical trial of metformin as addon treatment with clozapine in treatment of schizophrenia disorder. J. Res. Med. Sci. 20, 364-371 (2015)

22. Zhou, J. Q., Dong, Z. W., Zhou, X., Li, X. Z. \& Cun, J. Z. Metformin treatment of risperidone starting schizophrenia patients with the influence of bai metabolic control study: (In Chinese). Chin. J. Drug Abus. Prev. Treat. 20, 144-147 (2014). +154 .

23. Zhang, Y. C. The control study of metformin for olanzapine-induced metabolic dysfunction: (In Chinese). Med. J. Chin. People's Health 25, 69-70 (2013).

24. Jarskog, L. F. et al. Metformin for weight loss and metabolic control in overweight outpatients with schizophrenia and schizoaffective disorder. Am. J. Psychiatry 170, 1032-1040 (2013).

25. Chen, C.-H. et al. Effects of adjunctive metformin on metabolic traits in nondiabetic clozapine-treated patients with schizophrenia and the effect of metformin discontinuation on body weight: a 24-week, randomized, doubleblind, placebo-controlled study. J. Clin. Psychiatry 74, e424-e430 (2013).

26. Carrizo, E. et al. Extended release metformin for metabolic control assistance during prolonged clozapine administration: a 14 week, double-blind, parallel group, placebo-controlled study. Schizophr. Res. 113, 19-26 (2009).

27. Baptista, T. et al. Metformin as an adjunctive treatment to control body weight and metabolic dysfunction during olanzapine administration: a multicentric, double-blind, placebo-controlled trial. Schizophr. Res. 93, 99-108 (2007).

28. Baptista, T. et al. Metformin for prevention of weight gain and insulin resistance with olanzapine: a double-blind placebo-controlled trial. Can. J. Psychiatry 51, 192-196 (2006).

29. Choi, Y. J. Efficacy of adjunctive treatments added to olanzapine or clozapine for weight control in patients with schizophrenia: a systematic review and meta-analysis. ScientificWorldJournal 2015, 970730 (2015).

30. Domecq, J. P. et al. Clinical review: drugs commonly associated with weight change: a systematic review and meta-analysis. J. Clin. Endocrinol. Metab. 100, 363-370 (2015).

31. Fiedorowicz, J. G. et al. Systematic review and meta-analysis of pharmacological interventions for weight gain from antipsychotics and mood stabilizers. Curr. Psychiatry Rev. 8, 25-36 (2012).

32. Ellinger, L. K., Ipema, H. J. \& Stachnik, J. M. Efficacy of metformin and topiramate in prevention and treatment of second-generation antipsychoticinduced weight gain. Ann. Pharmacother. 44, 668-679 (2010).

33. Mizuno, Y. et al. Pharmacological strategies to counteract antipsychoticinduced weight gain and metabolic adverse effects in schizophrenia: a systematic review and meta-analysis. Schizophr. Bull. 40, 1385-1403 (2014).

34. Silva, V. A. D. et al. Metformin in prevention and treatment of antipsychotic induced weight gain: a systematic review and meta-analysis. BMC Psychiatry 16, 341 (2016).

35. Siskind, D. J., Leung, J., Russell, A. W., Wysoczanski, D. \& Kisely, S. Metformin for clozapine associated obesity: a systematic review and meta-analysis. PLOS ONE 11, e0156208 (2016).

36. Zhuo, C. et al. Topiramate and metformin are effective add-on treatments in controlling antipsychotic-induced weight gain: a systematic review and network meta-analysis. Front. Pharmacol. 9, 1393 (2018).

37. Zimbron, J., Khandaker, G. M., Toschi, C., Jones, P. B. \& Fernandez-Egea, E. A systematic review and meta-analysis of randomised controlled trials of treatments for clozapine-induced obesity and metabolic syndrome. Eur. Neuropsychopharmacol. 26, 1353-1365 (2016).

38. Leucht, S. et al. A meta-analysis of head-to-head comparisons of secondgeneration antipsychotics in the treatment of schizophrenia. Am. J. Psychiatry 166, 152-163 (2009).

39. Carbon, M., Kane, J. M., Leucht, S. \& Correll, C. U. Tardive dyskinesia risk with first- and second-generation antipsychotics in comparative randomized controlled trials: a meta-analysis. World Psychiatry 17, 330-340 (2018).

40. Galling, B. et al. Efficacy and safety of antidepressant augmentation of continued antipsychotic treatment in patients with schizophrenia. Acta Psychiatr. Scand. 137, 187-205 (2018). 
41. Baptista, T. et al. Metformin plus sibutramine for olanzapine-associated weight gain and metabolic dysfunction in schizophrenia: a 12-week double-blind, placebo-controlled pilot study. Psychiatry Res. 159, 250-253 (2008).

42. Wang et al. Metformin for treatment of antipsychotic-induced weight gain: a randomized, placebo-controlled study. Schizophr. Res. 138, 54-57 (2012).

43. Zhu, Y., Wang, Y. \& Wang, B. Efficacy of behavioral intervention therapy and metformin in the treatment of atypical antipsychotics-induced metabolic syndrome (In Chinese). J. Psychiatry 22, 195-197 (2009).

44. Higgins, J. \& Higgins, J. Cochrane handbook for systematic reviews of interventions. (Chichester, UK: John Wiley \& Sons, Ltd., 2008).

45. DerSimonian, R. \& Laird, N. Meta-analysis in clinical trials. Control. Clin. Trials 7, 177-188 (1986).

46. Egger, M., Davey Smith, G., Schneider, M. \& Minder, C. Bias in meta-analysis detected by a simple, graphical test. BMJ 315, 629-634 (1997).

47. Manna, V. On the essential role of omega-3 fatty acids in psychiatry. Ital. J. Psychopathol. 13, 222-242 (2007)

48. Jadad, A. R. et al. Assessing the quality of reports of randomized clinical trials: is blinding necessary? Control. Clin. Trials 17, 1-12 (1996).

49. Higgins, J. P. T., Green, S. \& Cochrane Collaboration. Cochrane handbook for systematic reviews of interventions (5.1.0 version). (Chichester, UK: The Cochrane Collaboration \& John Wiley \& Sons, Ltd., 2011).

50. Balshem, H. et al. GRADE guidelines: 3. Rating the quality of evidence. J. Clin. Epidemiol. 64, 401-406 (2011).
51. Atkins, D. et al. Grading quality of evidence and strength of recommendations. BMJ 328, 1490 (2004).

52. Sterne, J. A. et al. Recommendations for examining and interpreting funne plot asymmetry in meta-analyses of randomised controlled trials. BMJ $\mathbf{3 4 3}$, d4002 (2011).

53. Cohen, J. (ed) Statistical power analysis for the behavioral sciences 2 nd edn. (Hillsdale: Lawrence Erlbaum Associates, 1988)..

54. Cipriani, A. et al. Comparative efficacy and acceptability of 21 antidepressant drugs for the acute treatment of adults with major depressive disorder: a systematic review and network meta-analysis. Lancet 391, 1357-1366 (2018).

55. Erol, M., Bostan Gayret, O., Hamilcikan, S., Can, E. \& Yigit, O. L. Vitamin D deficiency and insulin resistance as risk factors for dyslipidemia in obese children. Arch. Argent. Pediatr. 115, 133-139 (2017).

56. van Stee, M. F., de Graaf, A. A. \& Groen, A. K. Actions of metformin and statins on lipid and glucose metabolism and possible benefit of combination therapy. Cardiovasc. Diabetol. 17, 94 (2018).

57. Maayan, L., Vakhrusheva, J. \& Correll, C. U. Effectiveness of medications used to attenuate antipsychotic-related weight gain and metabolic abnormalities: a systematic review and meta-analysis. Neuropsychopharmacology 35 , 1520-1530 (2010).

58. Alharbi, T. J. et al. The association of metformin use with vitamin B12 deficiency and peripheral neuropathy in Saudi individuals with type 2 diabetes mellitus. PLOS ONE 13, e0204420 (2018). 


\section{University Library}

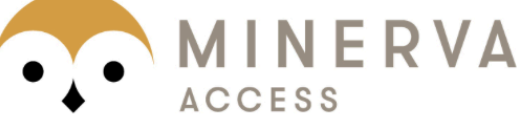

A gateway to Melbourne's research publications

Minerva Access is the Institutional Repository of The University of Melbourne

Author/s:

Jiang, W-L;Cai, D-B;Yin, F;Zhang, L;Zhao, X-W;He, J;Ng, CH;Ungvari, GS;Sim, K;Hu, ML;Zheng, W;Xiang, Y-T

Title:

Adjunctive metformin for antipsychotic-induced dyslipidemia: a meta-analysis of randomized, double-blind, placebo-controlled trials

Date:

2020-04-23

Citation:

Jiang, W. -L., Cai, D. -B., Yin, F., Zhang, L., Zhao, X. -W., He, J., Ng, C. H., Ungvari, G. S., Sim, K., Hu, M. -L., Zheng, W. \& Xiang, Y. -T. (2020). Adjunctive metformin for antipsychoticinduced dyslipidemia: a meta-analysis of randomized, double-blind, placebo-controlled trials. TRANSLATIONAL PSYCHIATRY, 10 (1), https://doi.org/10.1038/s41398-020-0785-y.

Persistent Link:

http://hdl.handle.net/11343/244396

License:

CC BY 